\title{
Long-Term Outcomes After Single-Balloon Enteroscopy: Are They Any Different from Double-Balloon Enteroscopy for Vascular Lesions?
}

\author{
Lauren B. Gerson
}

Published online: 4 July 2013

(c) Springer Science+Business Media New York 2013

Obscure GI bleeding is uncommon, accounting for approximately $5 \%$ of all GI bleeding episodes [1]. With the advent of capsule endoscopy and introduction of balloon enteroscopy to the United States in 2004, endoscopic visualization of the small bowel has become feasible and successful. Double-balloon enteroscopy (DBE) is associated with diagnostic yields of approximately 50-60\%, which exceed $70 \%$ in patients with obscure gastrointestinal bleeding (OGIB) [2]. Performance of video capsule endoscopy (VCE) prior to deep enteroscopy is associated with increased diagnostic yields. In an updated metaanalysis, the yield for DBE was $75 \%$ when a prior VCE study was abnormal, but only $27 \%$ after a negative VCE examination [3].

Single-balloon enteroscopy (SBE) was subsequently introduced into US clinical practice in 2008 in an effort to reduce time and complexity of the double-balloon procedure. Instead of using a second balloon on the distal end of the enteroscope as an anchor when the overtube is advanced, the tip of the enteroscope is deflected during the SBE procedure, creating a "hook" which functions similarly to the second balloon on the DBE enteroscope. Although less data have been published regarding SBE outcomes compared with DBE, initial studies reported somewhat lower diagnostic yields, potentially due to decreased rates of total enteroscopy $[4,5]$. Higher total enteroscopy rates and therapeutic interventions with DBE compared to SBE were reported when the enteroscope balloon was removed from the Fujinon system [6]. Nevertheless, a subsequent randomized controlled trial did not confirm these results [7].

L. B. Gerson ( $\square)$

Stanford University School of Medicine, Stanford, CA, USA

e-mail: lgersonmd@yahoo.com
In this issue of Digestive Diseases and Sciences, Kushnir et al. [8] from Washington University School of Medicine performed a retrospective cohort study in order to determine long-term outcomes after SBE. While longterm outcomes studies have been performed for DBE and are discussed below $[9,10]$, this literature contribution is the first long-term outcomes assessment for SBE. Given the conflicting data regarding efficacy of SBE compared to DBE, the major question is whether recurrent bleeding rates differ post-SBE compared to published rates postDBE. In this study, the authors reviewed 147 SBE examinations performed for the evaluation of OGIB between 2008 and 2010, following $110(75 \%)$ patients for a mean of 24 months post-procedure. Patients who participated in the follow-up phone calls or visits were more likely to have undergone SBE with positive findings in the small bowel leading to endoscopic therapy compared with patients who were lost to follow-up post-enteroscopy (69 vs. $35 \%$, $p<0.001)$. Seventy percent of the patients had undergone VCE studies before the enteroscopy examination. Significant lesions in the small bowel including vascular, ulcerative, and/or suspected neoplasms were detected in $91 \%$ of the patients undergoing capsule endoscopy. A source of bleeding was identified in 95/147 (65\%) SBE examinations including vascular lesions in $54 \%$, ulcers or erosions in $5 \%$, and small bowel masses in $3 \%$. Endoscopic therapy was performed in $76(52 \%)$ patients, and an additional eight were referred to surgery. Recurrent OGIB occurred in 50/110 (45 \%) patients available for follow-up. The authors were unable to find any risk factors associated with recurrent OGIB including Charlson co-morbidity index score, although the number of patients with valvular heart disease (22\%) was small. Recurrent bleeding occurred overall in 31/76 (41\%) of patients with a source found on SBE, and in 19/34 (56\%) of patients with normal 
SBE examination $(p=\mathrm{NS})$. There were similar rates of rebleeding in patients with vascular lesions and those with normal enteroscopy examinations, but none of the patients with non-vascular lesions re-bled.

Based on these findings, the efficacy of SBE, when longterm outcomes are assessed, does not appear to differ from that of DBE. This finding is not surprising, since vascular lesions account for the majority of the pathologic findings detected on deep enteroscopy examinations. Based on natural history studies, approximately $50 \%$ of small bowel angiodysplastic lesions continue to bleed without endoscopic therapy [11, 12], and as discussed below, similar percentages of patients can demonstrate recurrent overt or occult bleeding despite the performance of endoscopic therapy. The reasons for re-bleeding can include missed lesions on initial examination, either related to depth of insertion or inability to visualize all lesions, or recurrence of vascular lesions. Risk factors associated with recurrence have included advanced age $>65$ years, the presence of multiple lesions, transfusion requirement prior to endoscopic therapy, and the presence of aortic stenosis and/or chronic renal failure [10, 13, 14].

As discussed in the accompanying article, four prior studies assessed outcomes post-DBE for vascular lesions in the literature to date $[9,10,15,16]$. Re-bleeding was consistently defined as the presence of overt bleeding, need for transfusions, and/or ongoing need for iron therapy in order to include patients both with obscure overt and obscure occult bleeding. Re-bleeding rates for vascular lesions were $44 \%$ in the Gerson 2009 study, $60 \%$ in the Shinozaki 2010 study, and $48 \%$ in the current SBE study ( $p=0.4, \chi^{2}$ analysis). For patients with normal exams, rebleeding rates were $42 \%$ in the Gerson study, $37 \%$ in the Shozaki study, and $56 \%$ in the SBE study $(p=0.3)$. Therefore the rate of recurrent bleeding for SBE does not appear to differ compared to DBE for vascular lesions. This finding does not come as a surprise, given the current knowledge regarding small bowel vascular lesions and their tendency for re-bleeding and/or recurrence despite endoscopic therapy. As discussed above, re-bleeding should be expected in patients with underlying conditions associated with the formation of angioectasiae. These findings raise questions about the utility of repeat endoscopic therapy in patients with small bowel vascular lesions, particularly in the setting of hereditary syndromes, such as Osler-Weber-Rendu disease. While this area deserves further study, an algorithm of initial endoscopic therapy for small bowel vascular lesions followed by medical therapy to prevent recurrence, may be a more costeffective option for these patients. Currently available and effective medical therapy for refractory small bowel angioectasiae include octreotide, either as subcutaneous injection two to three times daily or intramuscular administration monthly $[17,18]$, or oral administration of thalidomide, based on a recent randomized controlled trial demonstrating increased efficacy compared to oral iron therapy [19].

Of major interest is the finding that re-bleeding rates were similar to those for vascular lesions in patients with normal enteroscopy examinations. This finding raises the question of whether the examinations were normal because vascular lesions were missed, or if bleeding occurred due to pathology that was perhaps submucosal and not accessible by endoscopic means. Computed tomographic (CT) or magnetic resonance enterography examinations were not routinely obtained after normal enteroscopy examinations. In a recent study, patients with normal VCE examinations underwent subsequent CT enterography (CTE) with pathologic findings described in up to $50 \%$ of the cohort [20]. In a 2011 study, 58 patients with obscure GI bleeding underwent VCE and CTE examinations in a blinded fashion. The sensitivity of CTE exceeded VCE ( 88 vs. $38 \%$ ), due to the superior ability of CTE to detect small bowel neoplasms [21]. In addition to an enhanced capability for the detection of submucosal masses, enterography may also be able to visualize submucosal vascular lesions.

In summary, SBE and DBE appear to be equivalent in terms of long-term outcomes and re-bleeding rates for vascular lesions. As over $50 \%$ of these patients experience recurrent bleeding, future research should focus on optimal management, including the identification and potential therapy for associated co-morbid conditions, such as aortic valvular disease. Studies comparing the utility of repeat endoscopic therapy to medical therapy, including a conservative approach with repeat iron infusions, are warranted based on the above discussion.

\section{References}

1. Raju GS, Gerson L, Das A, et al. American Gastroenterological Association (AGA) Institute technical review on obscure gastrointestinal bleeding. Gastroenterology. 2007;133:1697-1717.

2. Gerson LB. Outcomes associated with deep enteroscopy. Gastrointest Endosc Clin N Am. 2009;19:481-496.

3. Teshima CW, Kuipers EJ, van Zanten SV, et al. Double balloon enteroscopy and capsule endoscopy for obscure gastrointestinal bleeding: an updated meta-analysis. J Gastroenterol Hepatol. 2011;26:796-801.

4. Ramchandani M, Reddy DN, Gupta R, et al. Diagnostic yield and therapeutic impact of single-balloon enteroscopy: series of 106 cases. J Gastroenterol Hepatol. 2009;24:1631-1638.

5. Upchurch BR, Sanaka MR, Lopez AR, et al. The clinical utility of single-balloon enteroscopy: a single-center experience of 172 procedures. Gastrointest Endosc. 2010;71:1218-1223.

6. May A, Farber M, Aschmoneit I, et al. Prospective multicenter trial comparing push-and-pull enteroscopy with the single- and double-balloon techniques in patients with small-bowel disorders. Am J Gastroenterol. 2010;105:575-581. 
7. Domagk D, Mensink P, Aktas H, et al. Single- vs. double-balloon enteroscopy in small-bowel diagnostics: a randomized multicenter trial. Endoscopy. 2011;43:472-476.

8. Kushnir VM, Tang M, Goodwin J, et al. Long-term outcomes after single-balloon enteroscopy in patients with obscure gastrointestinal bleeding. Dig Dis Sci. (Epub ahead of print). doi: 10.1007/s10620-013-2588-y.

9. Gerson LB, Batenic MA, Newsom SL, et al. Long-term outcomes after double-balloon enteroscopy for obscure gastrointestinal bleeding. Clin Gastroenterol Hepatol. 2009;7:664-669.

10. Shinozaki S, Yamamoto H, Yano T, et al. Long-term outcome of patients with obscure gastrointestinal bleeding investigated by double-balloon endoscopy. Clin Gastroenterol Hepatol. 2010;8: 151-158.

11. Lai LH, Wong GL, Chow DK, et al. Long-term follow-up of patients with obscure gastrointestinal bleeding after negative capsule endoscopy. Am J Gastroenterol. 2006;101:1224-1228.

12. Saurin JC, Delvaux M, Vahedi K, et al. Clinical impact of capsule endoscopy compared to push enteroscopy: 1-year follow-up study. Endoscopy. 2005;37:318-323.

13. Godino C, Lauretta L, Pavon AG, et al. Heyde's syndrome incidence and outcome in patients undergoing transcatheter aortic valve implantation. J Am Coll Cardiol. 2013;61:687-689.

14. Chalasani N, Cotsonis G, Wilcox CM. Upper gastrointestinal bleeding in patients with chronic renal failure: role of vascular ectasia. Am J Gastroenterol. 1996;91:2329-2332.
15. May A, Friesing-Sosnik T, Manner H, et al. Long-term outcome after argon plasma coagulation of small-bowel lesions using double-balloon enteroscopy in patients with mid-gastrointestinal bleeding. Endoscopy. 2011;43:759-765.

16. Samaha E, Rahmi G, Landi B, et al. Long-term outcome of patients treated with double balloon enteroscopy for small bowel vascular lesions. Am J Gastroenterol. 2012;107:240-246.

17. Bon C, Aparicio T, Vincent M, et al. Long-acting somatostatin analogues decrease blood transfusion requirements in patients with refractory gastrointestinal bleeding associated with angiodysplasia. Aliment Pharmacol Ther. 2012;36:587-593.

18. Brown C, Subramanian V, Wilcox CM, et al. Somatostatin analogues in the treatment of recurrent bleeding from gastrointestinal vascular malformations: an overview and systematic review of prospective observational studies. Dig Dis Sci. 2010;55:2129-2134.

19. Ge ZZ, Chen HM, Gao YJ, et al. Efficacy of thalidomide for refractory gastrointestinal bleeding from vascular malformation. Gastroenterology. 2011;141:1629.e1-1637.e4.

20. Agrawal JR, Travis AC, Mortele KJ, et al. Diagnostic yield of dual-phase computed tomography enterography in patients with obscure gastrointestinal bleeding and a non-diagnostic capsule endoscopy. J Gastroenterol Hepatol. 2012;27:751-759.

21. Huprich JE, Fletcher JG, Fidler JL, et al. Prospective blinded comparison of wireless capsule endoscopy and multiphase CT enterography in obscure gastrointestinal bleeding. Radiology. 2011;260:744-751. 